typographical error). Nevertheless, they are few, and the general tone of the text is one of technical correctness and authority. Yet, despite these undoubted virtues, there is something lacking: it is simply that the author has not succeeded in making the field as enthralling as he found it during his 24 years with the United Kingdom Atomic Energy Authority. Instead, one is left with the uneasy feeling that the book reads like a succession of the carefully worded, technically correct, but bland handouts favoured by public relations officers, particularly in the nuclear industry. The sense of excitement aroused by the initial discovery of fission is missing, as is any sense of the enormous technological achievement in harnessing nuclear power.
After all, the reason why nuclear power bears the brunt of the anti-technologists' onslaught is precisely because it is seen as the high technology, an almost unique amalgam of science and engineering. Yet none of this emerges. Everything is under control, doubts are smoothly assuaged and technology rules supreme.

Nevertheless, for the reader who is prepared to accept these limitations, the book provides an accessible and comprehensive survey of the nuclear scene at a level which invites a wide readership.

Malcolm C. Scott is Senior Lecturer in the Department of Physics, University of Birmingham, in charge of the MSc course on the physics and technology of nuclear reactors.

\title{
Life as we know it: does anyone else?
}

\section{Paul Davies}

\begin{abstract}
Life Beyond Earth: The Intelligent Earthling's Guide to Life in the Universe. By Gerald Feinberg and Robert Shapiro. Pp.480. (Morrow: 1980.) Hardback \$14.95; paperback \$7.95. Earth and Cosmos. By R. S. Kandel. Pp.270. (Pergamon: 1980.) Hardback \$30, £13.50; paperback $\$ 14.90, £ 6.95$.
\end{abstract}

Life Beyond Earth, proclaims the publisher's blurb, "is the result of a threeyear collaboration between a theoretical physicist and a biochemist ...". That sounds like a recipe for disaster, but the end product is remarkably coherent, if a little prolix. It is also somewhat pretentious, because it presents a range of ideas, varying from uncontentious standard biochemistry to the most outrageous and bizarre speculations, in a deceptively uniform and scholarly format.

It is, of course, the outrageous ideas that make this book such fun to read. The authors pin their colours firmly to the mast in Chapter 1: "We believe that most other attempts... have not been sufficiently imaginative in examining the possibilities for extraterrestrial life". To remedy this state of affairs, the reader is invited to consider the astonishing prospect of living creatures inhabiting the most unlikely of habitats - wallowing in the molten interior of the Earth, bobbing about inside the Sun, crawling over the surfaces of neutron stars, or floating serenely and nebulously in interstellar space. This is all good sci-fi stuff, but my main misgiving is that while the mere existence of a source of free energy and a mechanism for selforganization are certainly necessary conditions for the appearance of some form of life, can we really believe, as the authors assume, that they are sufficient?

A related problem to the happy prospect of a Universe teeming with exotic life forms is that it is far easier to believe in the evolution of intelligence, once life has actually gained a foothold, than in the initial appearance of living things. Yet if intelligence abounds in the cosmos, we would expect it to be conspicuous. Where is the alien technology, some of it presumably billions of years more advanced than ours? Unfortunately, Feinberg and Shapiro almost completely ignore the subjects of intelligence and alien communities, so do not attempt to face this central issue.

It would be misleading, however, to over-emphasize the bizarre in this book. The authors provide a valuable service in, for example, drawing attention to the distorted public image of the Viking experiments on Mars, popularly believed to have excluded the possibility of life there. In fact, as the authors' careful analysis shows, the results were ambiguous, and the experiments in any case left a lot to be desired in their underlying assumptions about Martian biology.

One can also be persuaded by their argument that the search for extraterrestrial life contributes hugely to the motivation for the general exploration of space. Public disappointment that space probes have not yet positively encountered even the tiniest form of life has contributed to the widespread disillusionment with these expensive enterprises. Yet the most likely abode for life in the Solar System Jupiter - has still to be closely explored.

Inevitably, for a book that tackles a subject for which no known subject matter yet exists, Life Beyond Earth contains liberal doses of philosophy. Specially singled out for attack are those whom the authors dub as "carbaquists" _. biochemical faint-hearts who believe that life based on the chemistry of carbon and water is the only possibility. As a physicist, the carbaquist viewpoint has always seemed to me chauvinistic; biochemists are doubtless more conservative.
Another delicate issue concerns the need for a clear definition of life. Superficial criteria such as the ability to reproduce, response to environment and so on are also displayed by systems that are normally regarded as inanimate, so Feinberg and Shapiro adopt a sort of global definition wherein life is manifested through the activity of a biosphere; that is, one should look to the highly organized and complex structure of interlocking organisms as an unambiguous indicator of life.

At the end of the day we are still left with the depressing fact that the discovery of extraterrestrial biospheres is unlikely to occur in the twentieth century, so all books of this sort really amount to little more than speculation, or at best educated guesses. Nevertheless, there is a real need for us to examine fresh perspectives of mankind and his place in the Universe. With that in mind, I found the book entertaining and generally informative.

In contrast to this ambitious work, Earth and Cosmos by Robert Kandel is more modest in aim though wider in scope. The author also provides a cosmic perspective of mankind by discussing in detail a whole range of physical processes that are relevant to the structure of the Earth and its immediate environment. This book is full of fascinating information, ranging from continental drift to the pollution of the atmosphere. There is an extensive discussion of both meteorology and climatology, and a detailed review of the Earth's motion. Earth and Cosmos makes good reading for non-specialists who want to know more about the planet we live on.

Paul Davies is Professor of Theoretical Physics at the University of Newcastle upon Tyne.

\section{Cosmic questions}

\section{Joseph Silk}

The State of the Universe. Edited by G.T. Bath. Pp. 199. (Oxford University Press: 1980.) £8.95, $\$ 24.95$.

DESPITE its obscure origin and intemperate past, the Universe has made a good recovery. Conditions have stabilized over the past several eons, and practically all observers now agree that a recession is with us for some time to come. However the long-term outlook remains controversial - some predict that the rate of inflation will eventually drop, heralding the onset of a long era of deflation; other forecasters, perhaps a majority, maintain that the inflation rate will hold steady into the remote future. A vocal minority even persist in finding evidence that the inflation rate is accelerating.

This situation must seem familiar to the student of economics. Likewise, cosmologists also have their differences. It 
may seem strange to the layman that experts should disagree on whether the Universe will perpetually continue to cool down, to attain inexorably the ultimate energy crisis, or whether it is destined to overheat and implode into a brilliant fireball, hotter and more intense than anything imaginable by human beings. The uncertainty arises because the currently available indicators are often contradictory; the cosmologist has considerable scope for rejecting evidence that may not fit his model, especially if the evidence is incomplete and tentative. A choice still remains between the "Heat Death" of the Universe and the "Big Squeeze".

Yet our knowledge of the Universe is increasing at an unprecedented rate. The availability of observatories in space has enabled astronomers to study the Universe over much of the electromagnetic spectrum that is inaccessible from ground level, from gamma-rays to infrared radiation. And modern technology has revolutionized ground-based observing. The caricature of the earnest astronomer peering through a telescope in a cold, dark observatory dome is a myth that has long been displaced.

The modern astronomer punches in coordinates on a computer console and views the stars on a video display in a brightly lit room crammed with sophisticated electronic instrumentation. The aim is to probe ever deeper into the mysteries of the Universe, by furthering our understanding of the structures within it, from stars to galaxies, alone and in their great clusters.

Astronomers are now able - albeit barely so - to trace images of ordinary galaxies so distant that their light was emitted before the Earth was formed. In principle, with sufficient information about such systems, one could directly infer their distances and decide whether the rate of expansion of the Universe has changed significantly over this time-scale. The future of the Universe could then be predicted unambiguously. It seems likely that within the next one or two decades, provided that governments continue to make the necessary commitment to science, advances in technology, both ground-based and in space, will enable detailed studies to be made of remote galaxies at the observable edge of the Universe.

The State of the Universe, based on the Wolfson College Lectures given at Oxford in the Spring of 1979 , provides a fascinating and highly readable account of several of the major developments that have taken place in astronomy and cosmology over the past decade. Eight leading scientists who have played key roles in many of the discoveries cover a diverse array of topics, ranging from G.E. Hunt on the exploration of the planets to D.W. Sciama on the origin of the Universe. Especially memorable is the contribution by $\mathrm{R}$. Penrose on black holes, for its lucid account of space-time singularities. M.J.
Rees writes on galaxies, their nuclei and their origin, and R.J. Tayler describes current views on the origin of the chemical elements. Hunt's beautifully illustrated chapter is one of the highlights of the book, providing a full and timely account of the newly acquired knowledge of the planets from Mercury to Jupiter. Remaining chapters include D.E. Blackwell on the stars as suns, K.A. Pounds on the X-ray Universe and F.G. Smith on new ways of seeing the Universe. Geoffrey Bath is to be commended on having co-ordinated an extraordinary lucid array of timely contributions from a group of the most eminent, and certainly among the busiest, of British astrophysicists.

On reading The State of the Universe one cannot fail to be impressed by the immense vistas that the explosive progress in astronomy has revealed. Much is poorly understood: indeed, a radically new approach to physics may be required if we are to discover the origin of the Universe, for example. But enough has fallen into place for us to be confident that our theories enable us to understand how much of the Universe entered into its present state. Prediction of its future evolution now becomes feasible. We can already do this for stars: it may not be long before enough data is at hand to determine definitively the future of the Universe, no less.

Joseph Silk is Professor of Astronomy at the University of California, Berkeley, and author of The Big Bang, published earlier this year by W.H. Freeman.

\section{Tuning in to astronomy}

\section{David W. Hughes}

Starseekers. By Colin Wilson. Pp.271. (Hodder and Stoughton/Doubleday: 1980.) £10.95, $\$ 15.95$.

Colin Wilson, the Colin Wilson who wrote The Outsider, Introduction to the New Existentialism, Strange Powers and a host of other titles, has now turned his attention to astronomy. He has written a fascinating account of how he thinks astronomers tick today, did tick in the past and should tick in the future, if, that is, they ever wish to solve the "fundamental question". What is the question? Bertrand Russell once said that the aim of philosophy is to understand the Universe. Wilson urges us to go a step further. The question is not just "what is the Universe?". It is "what is the purpose of the Universe?'. Purpose, a word that makes all good scientists shudder. How should we - dry, dusty, intellectual astronomers, tied to our telescopes, computers and respectable thought processes - delve into the understanding of the purpose of things? Wilson reveals his answer by frequent use of such phrases as "flashes of second sight", "supra-logical processes", "paranormal" and "intuitive insight". We are not told to abandon our scientific principles, we are simply encouraged to recognize that they are incomplete. There is always more than meets the eye.

The book divides into three sections. The first is a review of ancient cosmologies. Why should our ancestors build Stonehenge, what was the real purpose of the pyramids, did the ancient Egyptians know about the precession of the equinoxes? Wilson refers often to bicameralism and has obviously been much affected by Julian Jaynes's book The Origin of Consciousness in the Breakdown of the

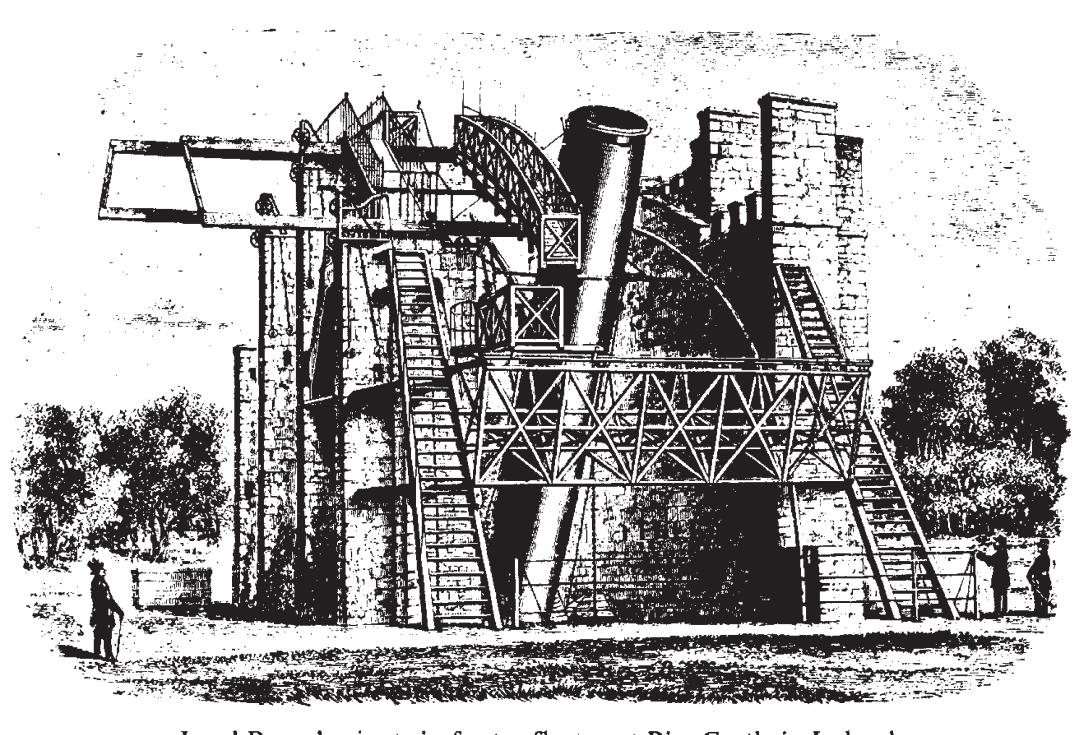

Lord Rosse's giant six-foot reflector at Birr Castle in Ireland 